\title{
Review
}

\section{An extension of fracture mechanics/technology to larger and smaller cracks/defects}

\author{
By Hiroyuki ABÉ*1,†
}

(Communicated by Takeo YokoBorI, M.J.A.)

\begin{abstract}
Fracture mechanics/technology is a key science and technology for the design and integrity assessment of the engineering structures. However, the conventional fracture mechanics has mostly targeted a limited size of cracks/defects, say of from several hundred microns to several tens of centimeters. The author and his group has tried to extend that limited size and establish a new version of fracture technology for very large cracks used in geothermal energy extraction and for very small cracks/defects or damage often appearing in the combination of mechanical and electronic components of engineering structures. Those new versions are reviewed in this paper.
\end{abstract}

Keywords: Fracture mechanics/technology, larger cracks, smaller cracks/defects, geothermal energy, optical glass fiber, electromigration failure

\section{Introduction}

Fracture mechanics is a subject of science-based engineering, and it is being used to assess the safety of engineering structures containing crack-like defects. Fracture mechanics is generally applied by equating combinations of stress and crack size to the measurement of resistance, which is geometrically independent, for crack extension.

The original work of the widely used fracture mechanics is the Griffith theory ${ }^{1)}$ which is extended and applied to metals. ${ }^{2), 3}$ ) An understanding of the strength and the fracture of materials, however, needs more interdisciplinary studies with their synthetical views. An outstanding and historical work was done by Professor Takeo Yokobori and his first book was published more than half a century ago.4) The author acknowledges that fracture mechanics is a subset of the Yokobori concept.

This paper is confined to fracture starting from cracks and/or defects. A great many papers on fracture mechanics have been published in the past several decades. Fracture mechanics has widely been ap-

*1 Professor Emeritus, Tohoku University, Miyagi, Japan.

$\dagger$ Correspondence should be addressed: H. Abé, Professor Emeritus, Graduate School of Engineering, Tohoku University, Aoba 6-601, Aoba-ku, Sendai, Miyagi 980-8579, Japan (e-mail: abe@abe. mech.tohoku.ac.jp). plied to the design of engineering structures and also for integrity assessments. The sizes of cracks in engineering structures appearing in those papers are mostly shown in the bold line in Fig. 1, say of from several hundred microns to at most several tens of centimeters. Two of our major studies are shown in Fig. 1. However these studies will not be reviewed since our major interest of this review is outside the bold line in Fig. 1.

A typical example for a large crack problem is the geothermal energy extraction from hot, dry rock mass. The cracks are artificially induced for use as heat exchange surfaces, through which water is circulated from the ground surface to the underground rock (Fig. 1). The sizes of cracks considered are as long as several kilometers. This discipline is referred to as Crustal Rock Fracture Mechanics.

The fracture phenomenon of a minute part of engineering structures is generally difficult to evaluate using the bulk material. The first example is a lifetime prediction of an optical glass fiber. The treated optical glass fiber has a diameter of $125 \mu \mathrm{m}$. A stable crack may extend perpendicular to the fiber axis when it is sufficiently small and extend in an unstable manner when it grows to its critical size. The crack usually is microns to several tens microns in size.

Engineering structures of today generally include 


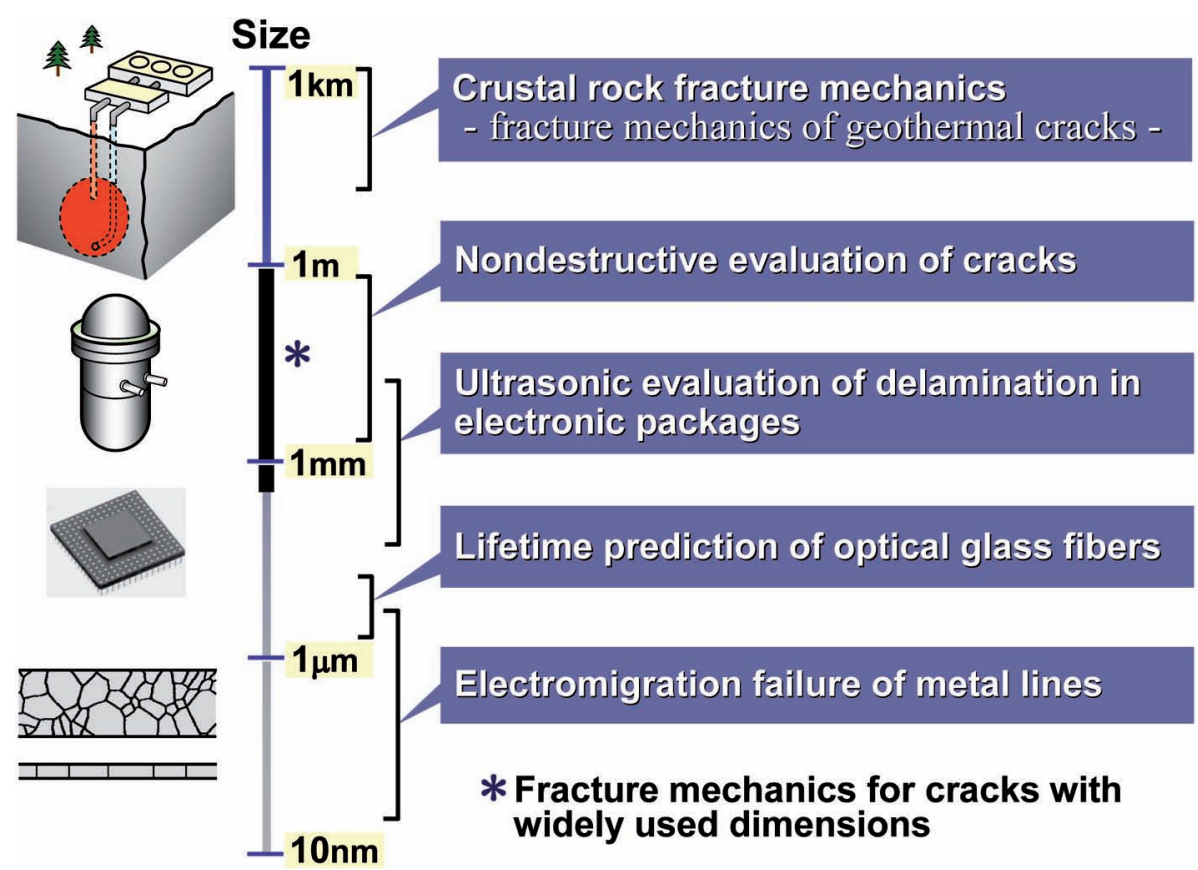

Fig. 1. Crack/defect size with wider range and Fracture Technology.

the combination of a mechanical and electronic and/ or microelectronic components. The fracture phenomenon in such components is much different from those for cracks shown in the bold line. As a typical example, a series of studies, which are part of the frontiers of fracture technology, on the electromigration failure of metal lines, will be cited.

\section{Crustal rock fracture mechanics}

It has been recognized that geothermal energy has the potential to be an alternative energy to fossilfuel. It is renewable and is almost free from global environmental problems such as global warming. In addition to these advantages, the estimated amount of geothermal energy in the earth's crust is abundant. Thus the establishment of highly reliable technology for recovering the abundant geothermal energy is critical for solving the global energy problems.

Obviously, the primary factors governing productivity of geothermal heat extraction are high temperature, existence of sufficient geothermal fluid and path of fluid-flow. However, fields with conditions satisfying all these three factors are rare. In this sense, in addition to the conventional type of geothermal heat extraction, advanced types of geothermal energy extraction, which are less dependent on natural subsurface conditions, are desirable for geothermal energy to be more promising. Hot Dry Rock (HDR) seems to be a candidate with the most potential among other advanced types. An HDR geothermal heat extraction subsurface system consists basically of a large artificial fracture (crack) with inlet and outlet wells through which water is heated and circulated back to the ground level (Fig. 1). Thus, HDR does not require natural geothermal fluid. An abundant and almost pollution-free energy will be made available by establishing a reliable methodology for creating the subsurface system as sketched in Fig. 1.

The concept of extracting heat from HDR was first proposed by a group at the Los Alamos Scientific Laboratory. The demonstration HDR project was made as a proof of concept in the 1970's.

The first step of the design of HDR geothermal systems was to form an artificial crack by hydraulic fracturing. The possibility of the formation of an artificial crack, after assuming that it is penny-shaped, was established analytically by means of a combination of fracture and fluid mechanics.5) It was also found that the extension of crack was essentially stable.

For the scientific promotion of the HDR geothermal extraction, a project named the $\Gamma$ project was set up in the Tohoku University.6) The project was 
awarded a Toray Science and Technology Grant in 1981, and was then adopted as a Specially Promoted Distinguished Research Program which was supported from 1983 to 1988 by the Ministry of Education, Science, Sports and Culture (MESSC, now MEXT). Some major results are shown in the following.

In the actual hydraulic fracturing, crack growth is difficult to observe directly. Therefore the hydraulic fracturing experiment using a large rock specimen (10.4 $\mathrm{m} \times 9.3 \mathrm{~m} \times 8.8 \mathrm{~m}$, Iidate granite) was performed at a quarry in Fukushima prefecture. ${ }^{7)}$ It was called Phase I of the $\Gamma$ project. The experiment using such a large sized specimen may help bridge the significant gap between the behaviors of small scale laboratory specimens and those of the actual subsurface rock mass.

After the fracturing, the huge rock specimen was cut along the crack plane. The specimen was the largest in the world in which the induced crack was observed directly. The crack propagation resistance was also obtained and was found to be larger than the resistance obtained from small specimens in the laboratory.

The next phase, Phase II, was the design of a subsurface system. In order to apply the results of Phase I, the subsurface cracks had to be provided in the rock mass at a reasonably shallow depth. A model field was selected at Higashi Hachimantai in Japan. In the field, a single fracture around $60 \mathrm{~m}$ in diameter was created at a depth of $380 \mathrm{~m}$ in jointfree, highly welded tuff based on rock fracture mechanics. The extension of the crack was largely influenced by the tectonic stress state. A method for measuring 3-D in-situ stress was newly developed by using massive fracturing techniques. ${ }^{8)}$ Thus the subsurface circulation system (inlet well - artificial crack - outlet well) was successfully constructed, as sketched in Fig. 1, and the design concept was validated. It was televised by NHK as a news flash (October 7, 1986).

Although the projects for Phase I and Phase II are sure to open up frontiers of rock fracture mechanics, the actual rock mass for geothermal energy extraction is still different, since the rock has numerous

*) HDR is defined as the extraction of heat from low permeability rocks possessing no significant natural fracture while HWR is the exploitation of geothermal heat by modification of the existing, but low permeability, natural fracture system. ${ }^{11)}$ micro-fractures under high temperature and/or high confining pressure. So in Phase III, rock properties were investigated under simulated geothermal conditions. ${ }^{9)}$

The main research concerns were:

(a) fracture growth behavior

(b) stress-corrosion cracking

(c) chemical water/rock interaction (dissolution and decomposition)

Figure 2 shows a part of the simulation.

One of the results is given in Fig. 3, where $\Delta \mathrm{a}$ is the crack extension and $\mathrm{K}(\mathrm{J})$ is the stress intensity factor calculated from the $J$-integral. It was the first investigation for rocks under the hydrothermal environment which showed a significant decrease in the crack propagation resistance.

Although the $\Gamma$ project may have given some remarkable scientific achievements, an actual reservoir is much deeper and more complicated in the geological structure even if an appropriate rock mass is selected. In order to apply the scientific results, including those of the $\Gamma$ project, we presented engineering systems fundamentals for the design concept and the design methodology for artificial geothermal reservoir systems. ${ }^{10)}$

There have been many HDR demonstration projects all over the world. This paper will not review a comparison of those demonstration projects but will briefly review the Hijiori project, which was sponsored by the New Energy and Industrial Technology Development Organization (NEDO), in Yamagata prefecture. The depth of the test field was about $2,300 \mathrm{~m}$ where the temperature was more than $270^{\circ} \mathrm{C}$. The type of the geothermal energy is somewhat different from the so-called HDR and is classified as HWR or HDR/HWR type.*) The Hijiori project put forth a geothermal energy extraction as an engineered system. The project was completed, and its outcome and technological issues for the future were summarized. ${ }^{12)}$

The first major academic meetings on HDR/ HWR were held in Japan. ${ }^{13)}$ The seminar was mainly sponsored by the National Science Foundation (NSF) in the United States and the Japan Society for the Promotion of Science (JSPS) in Japan. It was followed by a post-seminar symposium which provided an excellent forum for scientific exchange between the two countries, the United States and Japan, together with France, Germany and Australia. The author was a chairperson of the meetings. 


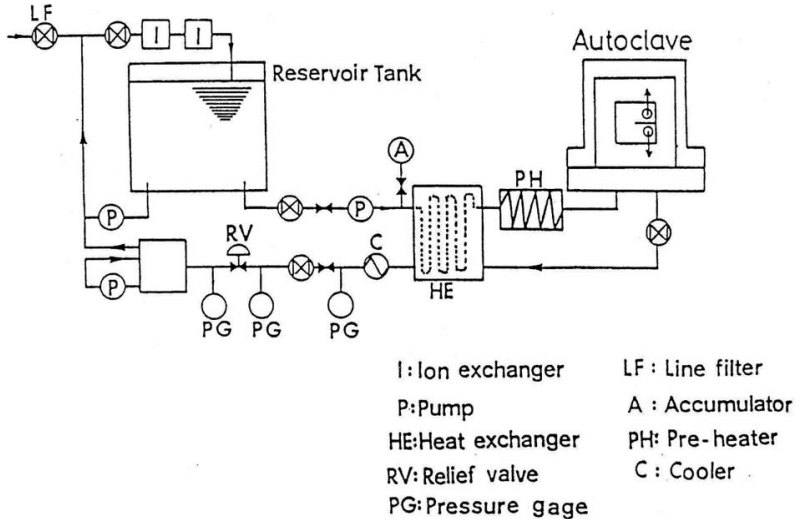

Fig. 2. Flow diagram of fracture toughness test under simulated geothermal conditions at temperatures up to $400^{\circ} \mathrm{C}$ and pressures up to $600 \mathrm{~kg} / \mathrm{cm}^{2}$. $\left.{ }^{9}\right)$

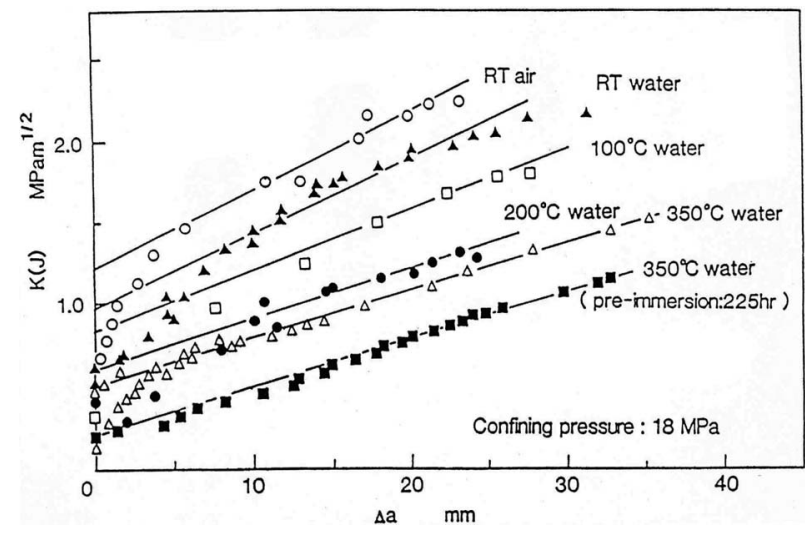

Fig. 3. Crack propagation resistance curves for Iidate granite under hydrothermal environments. ${ }^{9)}$

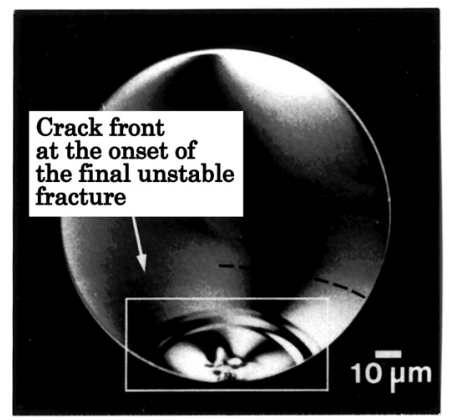

(A)
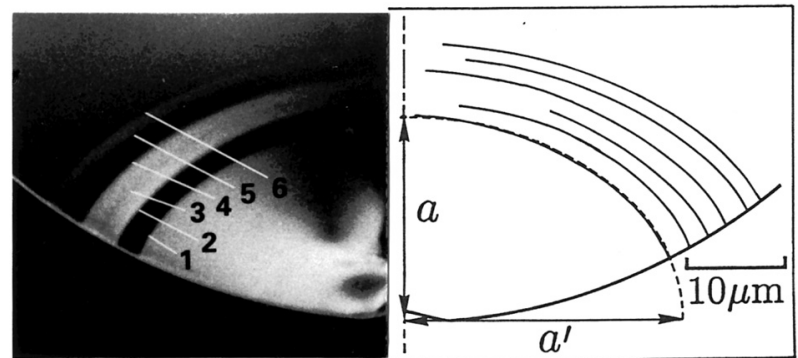

(B)

Fig. 4. Monograph of (A) the fractured surface of a silica optical fiber specimen $\left(25^{\circ} \mathrm{C}, 90 \% \mathrm{rh}\right)$, and (B) a closeup of the rectangular region in $\left.(\mathrm{A}) .{ }^{15}\right)$

In view of the many demonstration projects and taking stock of the progress that has been made world-wide after 13 years since the first meetings, a new international conference, in the form of a structured scientific/academic review, was held in Sendai. ${ }^{11)}$ It was sponsored by MESSC (now MEXT) and chaired by the author. The world's leading scientists and/or engineers from seven countries participated in this meeting. During the conference, a science and technology map was proposed for identifying the technologies and design paradigms which had been sufficiently developed for application to 


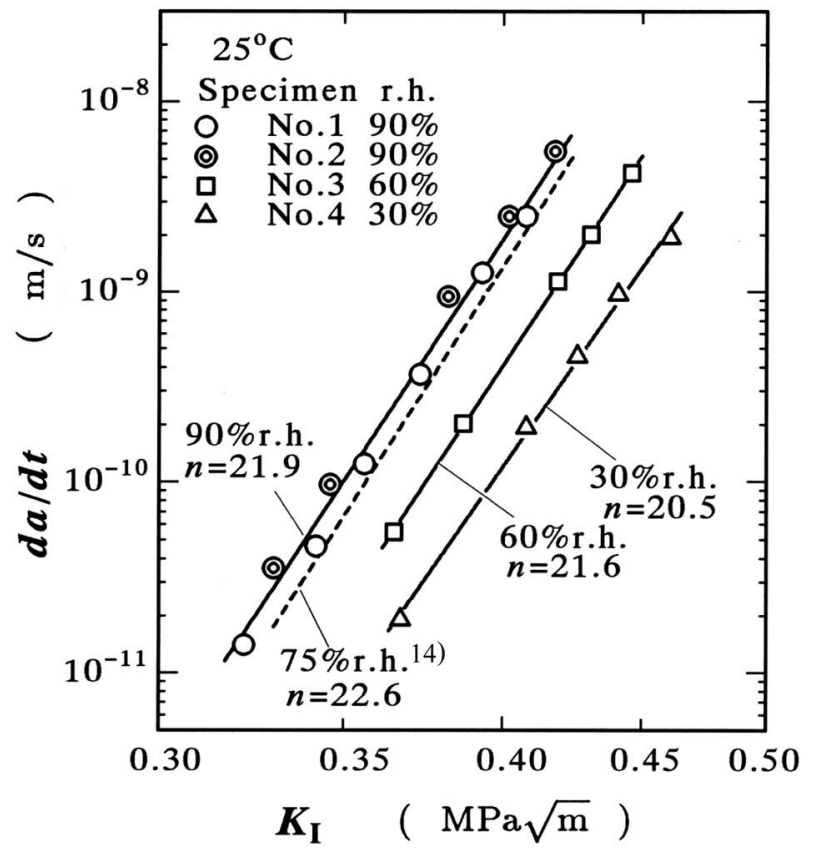

Fig. 5. Effect of humidity on the relation between the crack velocity $d a / d t$ and the stress intensity factor $K_{\mathrm{I}}$ for the optical fiber specimens. ${ }^{15)}$

commercial scale HDR/HWR operations, and those which require further development and fundamental research.

\section{Delayed failure in silica glass fibers}

As an example of fracture technology for cracks smaller than the bold line in Fig. 1, the lifetime prediction of optical glass fibers is first reviewed. A new method is developed for the direct measurement of subcritical small-crack growth in a silica optical fiber in our laboratory. ${ }^{14), 15)} \mathrm{A}$ monograph of a typical fractured surface is shown in Fig. 4. The repeatability of the method is found to be so excellent that the data obtained are considered to be very accurate compared with the other techniques proposed so far. The crack sizes measured are from 20 to $46 \mu \mathrm{m}$. Some of the results are summarized as follows:

(1) The data of the crack velocity $d a / d t$ can be fitted by a power function of the stress intensity factor $K_{\mathrm{I}}$ for a limited range of the crack velocity. The power $n$ is known as the stress-corrosion susceptibility constant.

(2) The crack velocity at a constant of $K_{\mathrm{I}}$ varies with relative humidity to a power of 2.5 . The

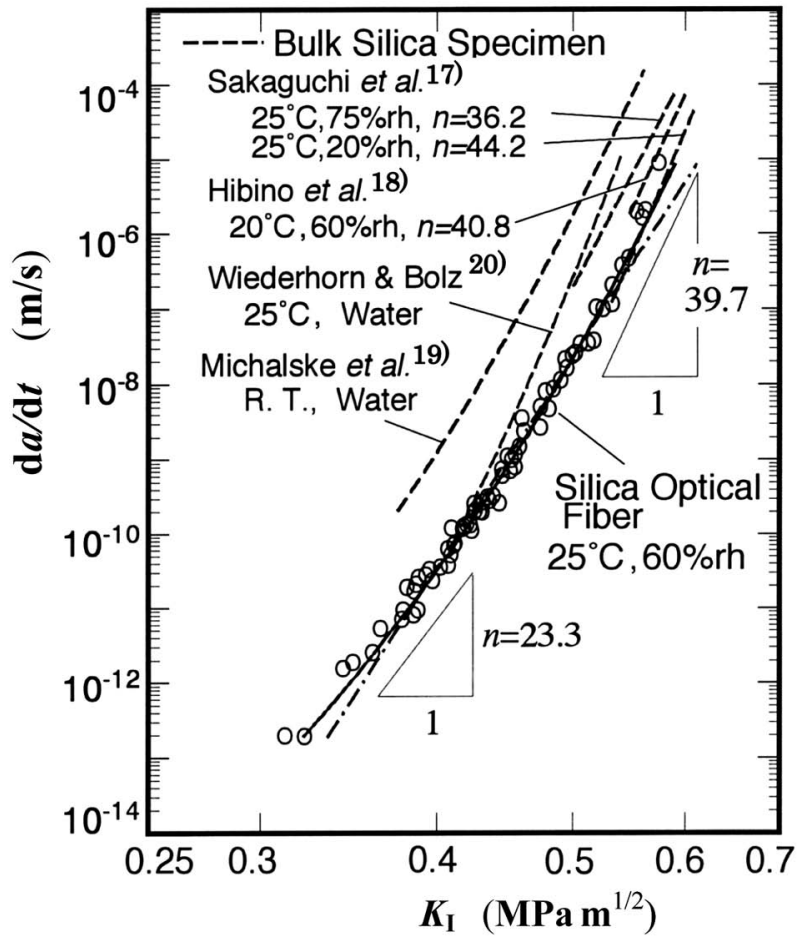

Fig. 6. Comparison of crack growth data for silica optical fibers and bulk silica specimen. The present data are plotted for annealed fiber specimens (o). Solid curve and chained lines represent least squares fits of the exponential law and the power law, respectively. Broken lines represent results for bulk silica specimens having large cracks. ${ }^{16}$ )

humidity dependence of the crack velocity is significant as shown in Fig. 5. ${ }^{15)}$

(3) Figure 6 shows the difference from the results for bulk silica specimens for a wide range of crack velocities. ${ }^{16)}$ The data agreed with the exponential law rather than the power law for a wide range of $d a / d t$. The effect of annealing is also shown.

As mentioned, most of engineering structures have been using the combination of mechanical and electronic components. The First Joint ASME/JSME Conference on Electronic Packaging was held in Milpitas, USA in 1992, where ASME stands for the American Society of Mechanical Engineers and JSME the Japan Society of Mechanical Engineers. The author, as Chair from JSME, received an ASME award at the Conference for his valued services which was complimented as a milestone for the electronic packaging industry. He also received an 

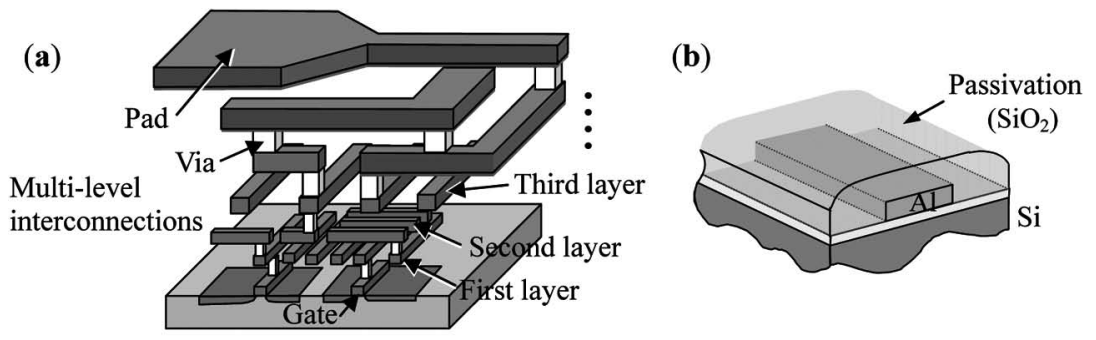

(c)

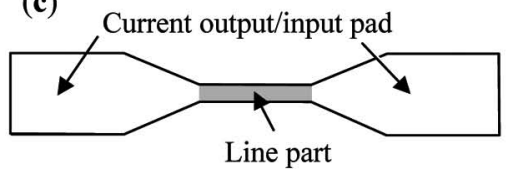

(d) Metal line 2 (Al)

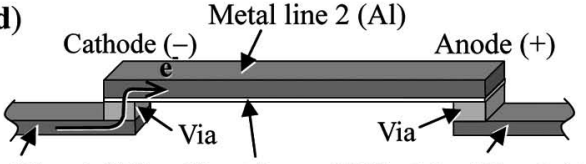

(e)

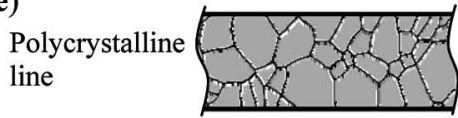

(f)

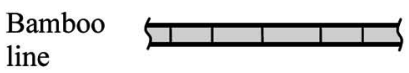

Fig. 7. Metal line structures treated. (a) Multi-level interconnections, (b) Passivated line, (c) Line connected with pads, (d) Via-connected line, (e) Polycrystalline lines, (f) Bamboo line. ${ }^{21)}$

Table 1. Comparison between conventional methods and the AFD-based method ${ }^{21)}$

\begin{tabular}{l} 
Conventional methods \\
\hline Lifetime prediction \\
Empirical equation ${ }^{23)}$ \\
Simple \\
Not universal (Many and long term experiments are \\
necessary for the respective line shapes even if the lines are \\
made of the same metallic films) \\
Inaccurate (It is difficult to predict a phenomenon in \\
operating condition from that in accelerated condition)
\end{tabular}

\section{Failure location}

Numerical simulation ${ }^{26)}$

Main purpose is to clarify the damage mechanisms

Method for determining the film characteristic constants of the line to be predicted is not developed

$\rightarrow$ Not necessarily suitable for quantitative failure prediction

\section{Evaluation of threshold current density $*, j_{t h}$}

Assumption of product of threshold and line-length being constant $^{27)}$

Simple and easy

Effect of line shape on $j_{t h}$ is not considered (Application is limited to only straight line)

The constant depends on temperature
Present method using $A F D$

AFD-based simulation of failure process ${ }^{30)-33)}$

Universal (Once the film characteristic constants are obtained, the failure prediction of any shaped line is possible under arbitrary operating conditions)

Accurate prediction for not only lifetime but also failure site

\section{AFD-based simulation of electromigration} behavior 22),34),35)

$A F D$ corresponds with actual amount of damage

Film characteristic constants can be derived by simple experiments to measure the amount of damage

AFD-based simulation for building-up process of atomic density distribution (incubation period) ${ }^{36}$

Universal and accurate (Once the film characteristic constants are obtained, the evaluation of the threshold in any shaped line is possible under arbitrary temperature)

$* j_{\text {th }}$ does not exist in case of metal lines with pads. 


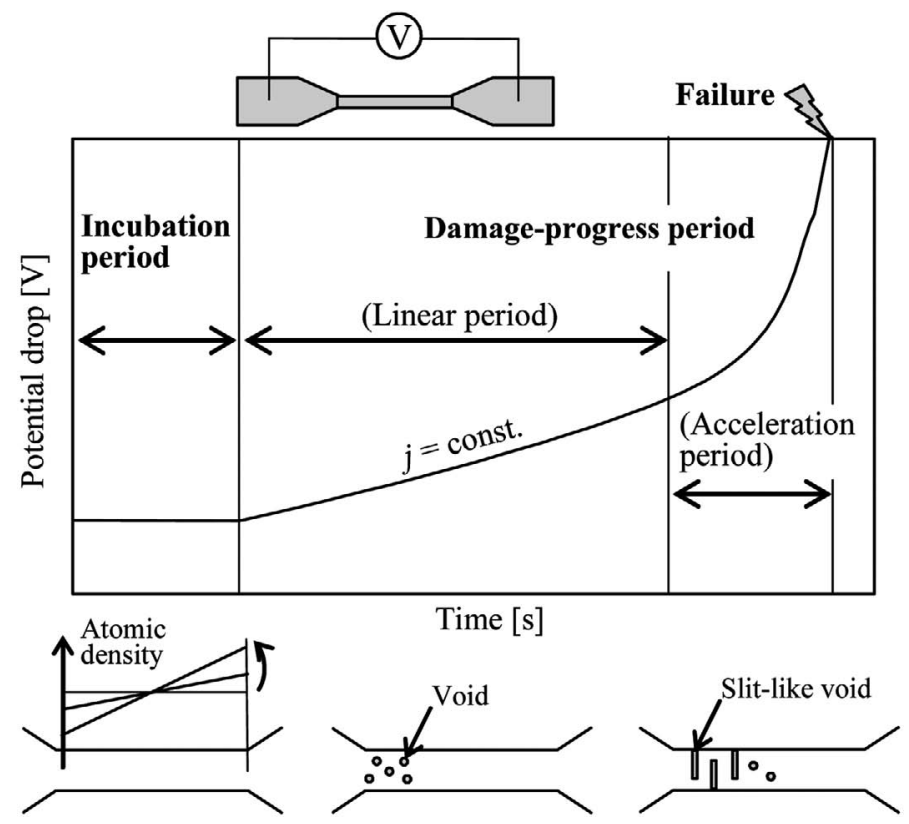

Fig. 8. Schematic diagram of the failure process. ${ }^{21)}$

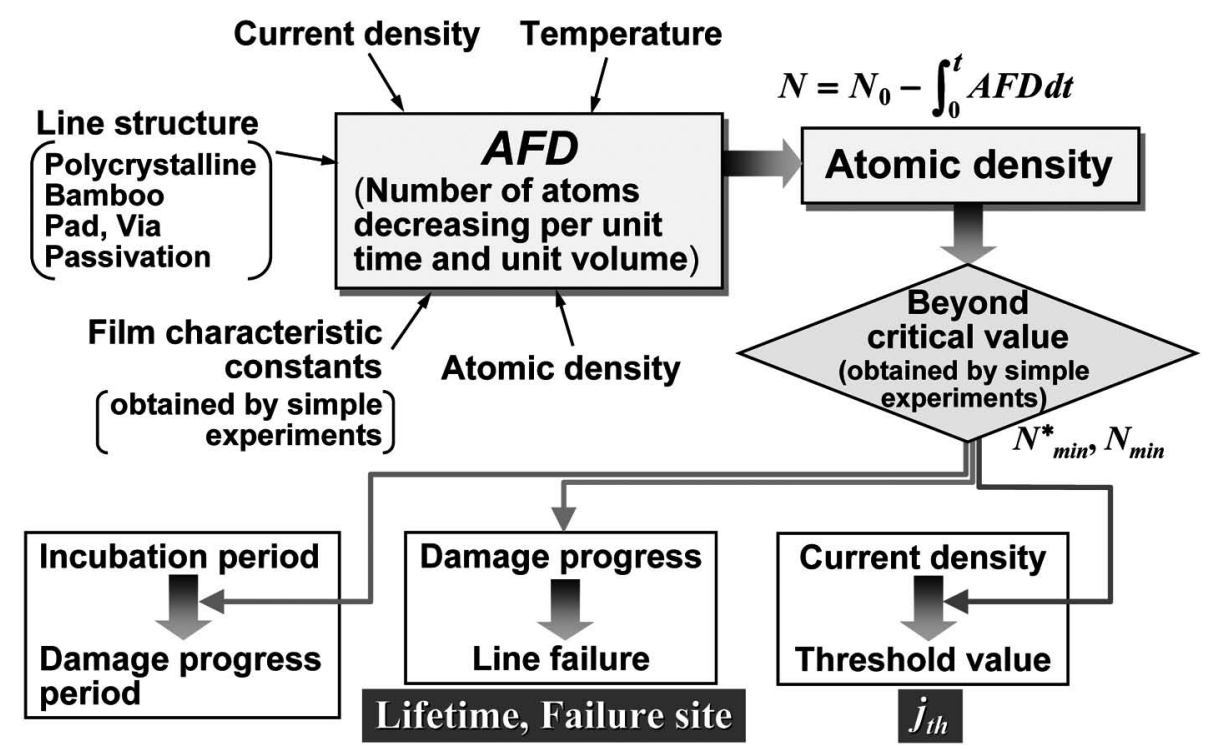

Fig. 9. Summary of $A F D$-based method, where $N^{*}$ is the atomic density in terms of the orientation of the microstructure and $N^{*}{ }_{\text {min }}$ is the critical atomic density for void initiation. ${ }^{21)}$

ASME Electrical and Electronic Packaging Division award in 1994 and an ASME Materials Division award for his assistance in organizing the Symposia on Materials and Mechanics of Electronic Packaging.

Since the 1992 Conference, in particular, many scientists and engineers have worked on the mechan- ical and electronic problems of minute components and excellent studies on reliability and prognostics have been published.

\section{Electromigration failure of metal lines}

Many engineering components integrate micro- 


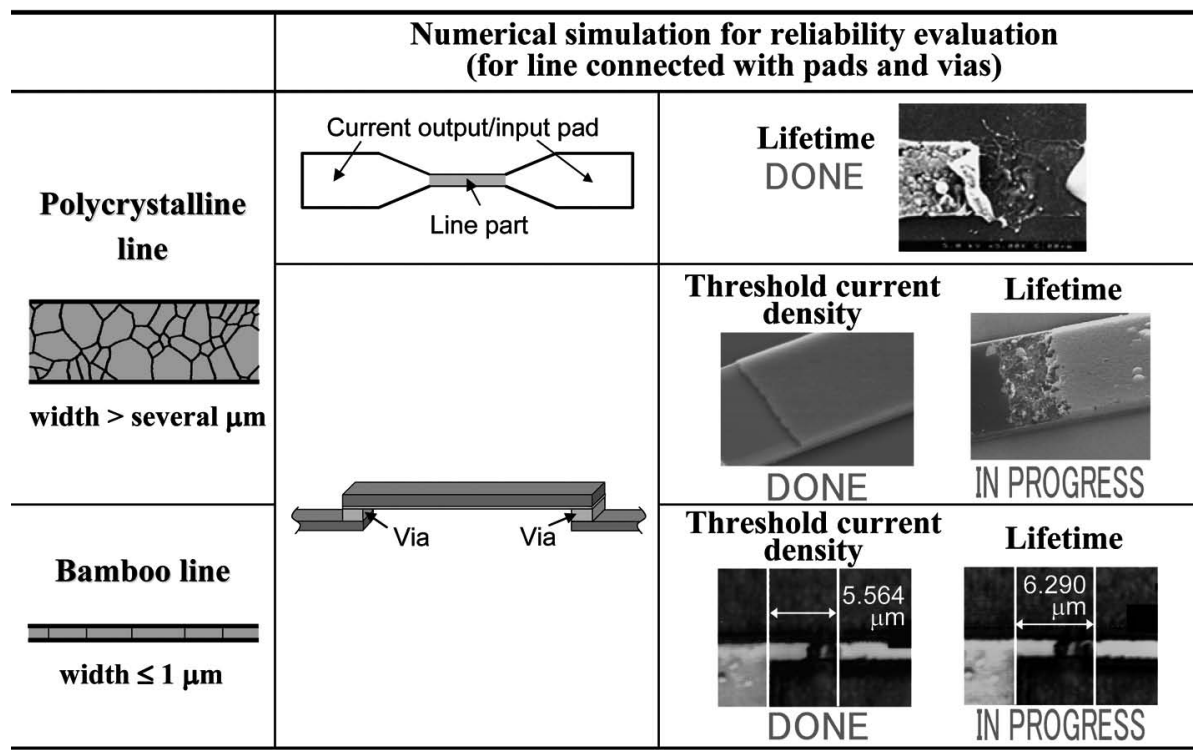

Fig. 10. Lifetime prediction for line connected with pads and evaluation of threshold current density $j_{\text {th }}$ for via-connected line. ${ }^{21)}$

circuits with finer and finer interconnecting metal lines. With the scaling down process, the density of electric current in the metal line increases, and the temperature of the microcircuit itself rises. Due to the trends of the operating conditions, such as high current density and high temperature, it is anticipated that metal line failure due to electromigration will become more serious now and in the future. Electromigration is a phenomenon where metallic atoms composing the line are transported by electron wind. The damage induced by electromigration appears as the formation of voids and hillocks. The growth of voids in the metal line ultimately results in electrical discontinuity as well as its final fracture. Therefore from the viewpoint of ensuring the reliability of integrated circuits (ICs), the lifetime of metal line must be predicted accurately. The damage is considered to be generated not by the so-called cracks but by defects induced by voids.

Multi-level interconnections in IC are illustrated in Fig. 7 (a). The metal lines used in the IC products are covered with a passivation layer as shown in Fig. 7(b), and the ends of the line are connected with large pads or vias for current input and output as shown in Figs. 7(c) and (d), respectively. Also, the microstructure of metal line distinguishes the socalled bamboo structured line from polycrystalline line depending on the size of metallic grains relative to the line width, see Figs. 7(e) and (f) for treated Al line.

The governing parameter is formulated based on the divergence of the atomic flux by electromigration, and is denoted by $A F D$, first introduced in 1998. ${ }^{22)}$ In other words, the parameter $A F D$ represents the number of atoms decreasing per unit time and unit volume. The $A F D$-based method makes it possible to predict the lifetime and possible failure site in universal and accurate way. The atomic density $N$ at any location within the metal line is calculated by

$$
N=N_{0}-\int_{0}^{t} A F D d t
$$

where $t$ is time and $N_{0}$ is the atomic density at a reference state. In actual calculations it is chosen as the state of stress-free and at $300 \mathrm{~K}$. The density $N$ is closely related to the initiation and growth of voids and hillocks.

Prior to the present studies, the prediction of the electromigration failure had been attempted by using an empirical equation ${ }^{23}$ and numerical simulations. ${ }^{24-26)}$ On the other hand, it is known that there is a threshold current density of electromigration damage, $j_{t h}$, below which no electromigration damage 
appears in the case of the via-connected metal lines. Conventionally, the threshold value has been evaluated based on the assumption that the product of the threshold and line-length is constant. ${ }^{27), 28)}$ The $A F D$-based method has resolved key problems inherent in the conventional methods as summarized in Table 1.

Presently the width of metal lines ranges from several tens $\mathrm{nm}$ to several tens $\mu \mathrm{m}$. In this study, the lines of $980 \mathrm{~nm}$ to $9.9 \mu \mathrm{m}$ in width are considered. The line width of around $1 \mu \mathrm{m}$ gives the transition between polycrystalline and bamboo types.

Lifetime and possible failure location are predicted by means of the numerical simulation of the failure process, covering the build up of atomic density distribution, void initiation, void growth and ultimately line failure in the incubation and damage process as shown in Fig. 8.

The AFD-based method developed in our laboratory is summarized in Fig. 9. The governing parameter $A F D$ integrates all the factors affecting the damage, i.e., the line structure, film characteristics, operating conditions such as current density and temperature, and atomic density.

By utilizing $A F D$ the distribution of atomic density $N$ within the metal line can be calculated from [1]. If the atomic density is beyond a critical value, the incubation period evolves into the damage-progress period and the threshold current density $j_{t h}$ is measured. An excess of the atomic density over the critical value is used for changing the damage-progress to line failure and lifetime and possible failure site are predicted.

For the polycrystalline line connected with pads, the parameter $A F D$ has been identified in the passivated polycrystalline line. Furthermore, the thickness of the passivation layer for the required lifetime of the line has been developed.

Respecting the polycrystalline line connected with vias, the governing parameter $A F D$ at the ends of the passivated polycrystalline line has been identified. The evaluation method of the threshold current density has been developed. It has been shown that the evaluation result of threshold current density in the passivated polycrystalline line agreed with the experimental one, and the usefulness of the $A F D$ based method has been confirmed.

As to the bamboo structured line connected with vias, the governing parameter $A F D$ in the passi- vated bamboo line has also been identified. The evaluation method of the threshold current density for the passivated bamboo line has also been developed by using $A F D$. The usefulness of the $A F D$-based method has been shown experimentally.

These results are described again in Fig. 10.

As a future prospect of the development of reliability evaluation method, the followings are noted:

(1) The reliability evaluation method is expected to be applied to the line structure of $\mathrm{Cu}$ metallization.

(2) When we consider the line-width from $980 \mathrm{~nm}$ treated here down to several tens of $n m$, it is still an open question and interesting problem how our approach including experimental technique can be effective for the finer lines.

The paper (Ref. 21) was written out based on a plenary lecture presented at the 11th International Conference on Fracture (ICF 11), held in Turin, Italy, on March 20-25, 2005.

\section{Concluding remarks}

The research works shown in this review paper, which might be small number in the examples, are pioneering particularly in the extension of fracture mechanics/technology, where each of them is based on its own background on science and technology. Further refinements and challenges should of course be required, where the fracture is characterized not only by geometrical and mechanical effects but by thermal, electronic, chemical and/or physical effects depending on the research target. Fortunately, many other researchers particularly in the United States and Japan have recently tried to join in working on such new areas and excellent results have also been found in extending fracture mechanics/technology.

\section{Acknowledgements}

The author wishes to express his gratitude to Professor Takeo Yokobori, M.J.A. for his valuable advice. The author acknowledges all of the former research associates and graduate students of his group. The author wishes to express his thanks to the late Professor Hideaki Takahashi, Professors Katsuto Nakatsuka, Hiroaki Niitsuma and Tetsuo Shoji for their cooperation in particular in the experimental work of the $\Gamma$ project. Appreciation is also extended to Prof. Masumi Saka and Mr. Takao Shōji for their help with the preparation of the manuscript. 


\section{References}

1) Griffith, A. A. (1920) The phenomena of rupture and flow in solids. Phil. Trans. Roy. Soc. London, A221, 163-198.

2) Irwin, G. R. (1948) Fracture dynamics. In Fracturing of Metals (eds. Jonassen, F., Roop, W. P. and Bayless, R. T.). ASM, pp. 147-166.

3) Orowan, E. (1950) Fatigue and Fracture of Metals, MIT Press. Cambridge, MA.

4) Yokobori, T. (1955) Zairyo-Kyodo Gaku. Gihodo, Tokyo (Translated Edition (1965), The Strength, Fracture and Fatigue of Materials, ed. Crisp, J. D. C., Monash Univ., translated by Matsuo, S. and Inoue, M., P. Noordhoff, Groningen, The Netherlands).

5) Abé, H., Mura, T. and Keer, L. (1976) Growth rate of a penny-shaped crack in hydraulic fracturing of rocks. J. Geophys. Res. 81, 5335-5340.

6) Abé, H. and Takahashi, H. (1983) Crustal rock fracture mechanics for design and control of artificial subsurface cracks in geothermal energy extraction engineering ( $\Gamma$-project). Proc. 9th Workshop Geothermal Reservoir Engineering, Stanford, California, December, 1983, pp. 415-420.

7) Abé, H., Hayashi, K. and Hashida, T. (1989) Studies on crack propagation resistance of rocks based on hydrofrac data of large specimens. SEM/RILEM Int. Conf. on Fracture of Concrete and Rock, Houston, Texas, June, 1987 (eds. Shah, S. P. and Swartz, S. E.), Springer-Verlag, New York, pp. 354-361.

8) Hayashi, K., Shoji, T., Niitsuma, H., Ito, T. and Abé, H. (1985) A new in-situ tectonic stress measurements and its application to a geothermal model field. Geothermal Resources Council, Trans. 9, 99-104.

9) Takahashi, H., Shoji, T. and Abé, H. (1987) Recent progress and future of " $\Gamma$ "-project at Tohoku University, Japan. Geothermics 16, 409-418.

10) Abé, H. and Hayashi, K. (1992) Fundamentals of design concept and design methodology for artificial geothermal reservoir systems. Geothermal Resources Council Bulletin 21, 149-155.

11) Structured Academic Review of HDR/HWR - Engineered Geothermal Systems, How We Think Reservoirs Work And What To Do Next - March 14-16, 1997, Tohoku University, sponsored by Tohoku Univ. and MESSC (now MEXT), chaired by Abé, H.

12) National Institute of Advanced Industrial Science and Technology (2004) Current status and prospect of engineered geothermal systems: Reviews of Hijiori HDR Project, AIST report (eds. Niitsuma, H. et al.) (in Japanese).

13) Hydraulic Fracturing and Geothermal Energy (1983) Proc. First Japan-United States Joint Seminar on Hydraulic Fracturing and Geothermal Energy, Tokyo, November, 1982, and Symp. on Fracture Mechanics Approach to Hydraulic Fracturing and Geothermal Energy, Sendai, November, 1982 (eds. Nemat-Nasser, S., Abé, H. and Hirakawa, S.), Martinus Nijhoff Publishers, The Hague, The Netherlands.

14) Muraoka, M. and Abé, H. (1991) A new approach to evaluate the delayed fracture behavior of an optical glass fiber. Proc. Mater. Res. Soc. Symp. 226, Anaheim, California, April, 1991 (eds. Suhir, E. et al.), MRS, pp. 369-374.

15) Muraoka, M., Ebata, K. and Abé, H. (1993) Effect of humidity on small-crack growth in silica optical fibers. J. Am. Ceram. Soc. 76, 1545-1550.

16) Muraoka, M. and Abé, H. (1996) Subcritical crack growth in silica optical fibers in a wide range of crack velocities. J. Am. Ceram. Soc. 79, 51-57.

17) Sakaguchi, S., Sawaki, Y., Abe, Y. and Kawasaki, T. (1982) Delayed failure in silica glass. J. Mater. Sci. 17, 2878-2886.

18) Hibino, Y., Sakaguchi, S. and Tajima, Y. (1984) Crack growth in silica glass under dynamic loading. J. Am. Ceram. Soc. 67, 64-68.

19) Michalske, T. A., Smith, W. L. and Bunker, B. C. (1991) Fatigue mechanisms in high-strength silicaglass fibers. J. Am. Ceram. Soc. 74, 1993-1996.

20) Wiederhorn, S. M. and Bolz, L. H. (1970) Stress corrosion and static fatigue of glass. J. Am. Ceram. Soc. 53, 543-548.

21) Abé, H., Sasagawa, K. and Saka, M. (2006) Electromigration failure of metal lines. Int. J. Fracture 138, 219-240.

22) Sasagawa, K., Nakamura, N., Saka, M. and Abé, H. (1998) A new approach to calculate atomic flux divergence by electromigration. Trans. ASME, J. Electron. Packag. 120, 360-366.

23) Black, J. R. (1969) Electromigration failure modes in aluminum metallization for semiconductor devices. Proc. IEEE 57, 1587-1593.

24) Nikawa, K. (1981) Monte Carlo calculations based on the generalized electromigration failure model. Proc. 19th IEEE Int. Reliability Phys. Symp. Orland, Florida, April, 1981, IEEE, pp. 175-181.

25) Marcoux, P. J., Merchant, P. P., Naroditsky, V. and Rehder, W. D. (1989) New 2D simulation model of electromigration. Hewlett-Packard J., June, 79-84.

26) Kirchheim, R. and Kaeber, U. (1991) Atomistic and computer modeling of metallization failure of integrated circuits by electromigration. J. Appl. Phys. 70, 172-181

27) Blech, I. A. (1976) Electromigration in thin aluminum films on titanium nitride. J. Appl. Phys. 47, 1203-1208.

28) Oates, A. S. (1991) Electromigration in multilayer metallization: drift-controlled degradation and the electromigration threshold of $\mathrm{Al}-\mathrm{Si}-\mathrm{Cu} / \mathrm{TiN}_{\mathrm{X}} \mathrm{O}_{\mathrm{Y}} /$ $\mathrm{TiSi}_{2}$ contacts. J. Appl. Phys. 70, 5369-5373.

29) McPherson, J. W. (1986) Stress dependent activation energy. Proc. 24th IEEE Int. Reliability Phys. Symp., Anaheim, California, April, 1986, IEEE, pp. $12-18$.

30) Sasagawa, K., Naito, K., Saka, M. and Abé, H. (1999) A method to predict electromigration failure of metal lines. J. Appl. Phys. 86, 6043-6051.

31) Sasagawa, K., Hasegawa, M., Naito, K., Saka, M. and Abé, H. (2001) Effects of corner position and operating condition on electromigration failure in angled bamboo lines without passivation layer. Thin Solid Films 401, 255-266. 
32) Sasagawa, K., Hasegawa, M., Saka, M. and Abé, H. (2002) Prediction of electromigration failure in passivated polycrystalline line. J. Appl. Phys. 91, 9005-9014.

33) Sasagawa, K., Hasegawa, M., Yoshida, N., Saka, M. and Abé, H. (2003) Prediction of electromigration failure in passivated polycrystalline line considering passivation thickness. Proc. InterPACK'03 (CDROM), Maui, Hawaii, July, 2003, ASME, Paper ID IPACK 2003-35065.

34) Sasagawa, K., Hasegawa, M., Saka, M. and Abé, H. (2000) Atomic flux divergence in bamboo line for predicting initial formation of voids and hillocks.
Theoretical and Applied Fracture Mechanics 33, $67-72$.

35) Sasagawa, K., Hasegawa, M., Saka, M. and Abé, H. (2002) Governing parameter for electromigration damage in passivated polycrystalline line. J. Appl. Phys. 91, 1882-1890.

36) Hasegawa, M. (2004) Evaluation method of electromigration damage in IC metal lines and its application to practical problems, Ph. D. dissertation, Tohoku University, Sendai, Japan.

(Received Aug. 27, 2009; accepted Oct. 8, 2009)

\section{Profile}

Hiroyuki Abé was born in 1936. He received his B. Eng. and Ph. D. in Mechanical Engineering from Tohoku University. In 1977 he became Professor at Tohoku University. He had been Dean of School and Graduate School of Engineering (1993-1995) and President of Tohoku University (1996-2002). He was installed as Executive Member of the Council of Science and Technology Policy in the Cabinet Office (2003-2007). He is the Counselor to the President of the Japan Science and Technology Agency.

In 1975, 1985, 1992, 2000 and 2005 he received the JSME Medals from the Japan Society of Mechanical Engineers (JSME), in 1998 the Materials and Mechanics Award from the Materials and Mechanics Division and in 1999 the Bioengineering Award

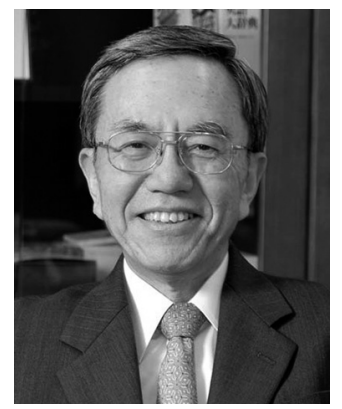
from the Bioengineering Division of the JSME, in 1979 the Best Paper Award from the Japanese Society of Medical Electronics and Bioengineering and in 1984 the Award from the Mining and Metallurgical Institute of Japan. He received in 1987 the Best Paper Award and in 1998 the Geothermal Research Award from the Geothermal Research Society of Japan. Also he received in 1991 and 2002 the Award from the Japanese Society for Non-Destructive Inspection. In 1992 the R. E. Peterson Award was presented to him for the best application paper in Experimental Mechanics from the Society for Experimental Mechanics (USA). He was also awarded BEST PAPER in 14th World Conference on NDT\&INTEXT NDT '96. In 2000 he received the Medal of Honor with Purple Ribbon from the Government of Japan. He was elected as Foreign Associate of the US National Academy of Engineering in 2002. 